\title{
BOUNDS FOR PERPETUAL AMERICAN OPTION PRICES IN A JUMP DIFFUSION MODEL
}

\author{
ERIK EKSTRÖM,* University of Manchester
}

\begin{abstract}
We provide bounds for perpetual American option prices in a jump diffusion model in terms of American option prices in the standard Black-Scholes model. We also investigate the dependence of the bounds on different parameters of the model.
\end{abstract}

Keywords: American option; bound; jump diffusion; optimal stopping

2000 Mathematics Subject Classification: Primary 91B28

Secondary 60G40

\section{Introduction}

In the standard Black-Scholes model, in which stock prices are modeled by geometric Brownian motion, the valuation of perpetual American options is well understood. Indeed, the value of a perpetual American option can typically be determined explicitly by solving the corresponding free boundary problem (see McKean (1965) for an early reference), or by finding the smallest concave majorant of the (appropriately transformed) payoff function (see Dayanik and Karatzas (2003)). However, if incorporating jumps into the stock price dynamics, then explicit formulae for the value of perpetual options are rare (see Mordecki (2002) for results on the American put option).

It is well known (see Alvarez (2003)) that prices of perpetual American options in diffusion models are monotonically increasing in the volatility. In this paper we provide bounds for American option prices in a jump diffusion model in terms of American option prices in a standard Black-Scholes model with a sufficiently large volatility.

In Section 2, we introduce the jump diffusion model. Then we state and prove our main result. It ensures the existence of a volatility so that the corresponding Black-Scholes price exceeds the jump diffusion price of any American option. Moreover, it characterizes the minimal such volatility. In Section 3, we investigate the dependence of the minimal volatility on different parameters of the jump diffusion model.

\section{The model and the main result}

To model the jumps of the stock price process, let $v$ be a Poisson random measure on $[0, \infty) \times[0,1]$ with intensity measure $\lambda \mathrm{d} t \mathrm{~d} z$ for some constant $\lambda \geq 0$, and let

$$
\tilde{v}(\mathrm{~d} t, \mathrm{~d} z):=v(\mathrm{~d} t, \mathrm{~d} z)-\lambda \mathrm{d} t \mathrm{~d} z
$$

Received 21 November 2005; revision received 9 May 2006.

* Postal address: School of Mathematics, University of Manchester, Sackville Street, Manchester M60 1QD, UK.

Email address: ekstrom@maths.manchester.ac.uk

The author thanks T. Iger for helpful discussions. 
be the corresponding compensated jump measure. Further, assume that $W$ is a standard Brownian motion which is independent of $v$, and let $\left(\mathcal{F}_{t}\right)_{0 \leq t<\infty}$ be the filtration generated by $W$ and $v$ augmented by null sets.

The stock price process $X$ is modeled as the unique solution to the stochastic differential equation

$$
\mathrm{d} X(t)=r X(t-) \mathrm{d} t+\sigma X(t-) \mathrm{d} W+X(t-) \int_{z=0}^{1} \phi(z) \tilde{v}(\mathrm{~d} t, \mathrm{~d} z) .
$$

Here the constant $\sigma \geq 0$ is the volatility of the continuous fluctuations of $X$, the constant $r>0$ is the interest rate, and $\phi:[0,1] \rightarrow[-1, \infty)$ specifies the possible relative jump sizes. The interpretation of the model is as follows. Jumps occur at the constant rate $\lambda \geq 0$. Associated to each jump of $X$ is a 'label' $z$. If a jump occurs at time $t$ with a label $z$, then the jump size is $\phi(z) X(t-)$. We assume that the label space is $[0,1]$ and that the distribution of labels is uniform. Note, however, that the label space and the label distribution can be made arbitrary upon a change of variables. Throughout this article we assume that the function $\phi$ is measurable and satisfies

$$
\int_{0}^{1}|\phi(z)| \mathrm{d} z<\infty
$$

and

$$
\int_{0}^{1}(1+\phi(z))^{\varepsilon} \mathrm{d} z<\infty
$$

for some $\varepsilon<0$.

Remark. The solution to (2.1) can be written more explicitly as

$$
X(t)=X(0) \exp \left\{\left(r-\frac{\sigma^{2}}{2}-\lambda \int_{0}^{1} \phi(z) \mathrm{d} z\right) t+\sigma W(t)+\sum_{k=1}^{N(t)} Y_{k}\right\},
$$

for some sequence $\left\{Y_{k}\right\}_{k=1}^{\infty}$ of independent and identically distributed random variables, where $N(t):=\int_{t=0}^{t} \int_{z=0}^{1} v(\mathrm{~d} t, \mathrm{~d} z)$ is a Poisson process with intensity $\lambda$. The distribution of the $Y_{k} \mathrm{~s}$ is connected with the specification of $\phi$ through the relation

$$
\mathrm{P}\left(Y_{k} \leq a\right)=\mu\left(\left\{z \in[0,1]: \phi(z) \leq \mathrm{e}^{a}-1\right\}\right),
$$

where $\mu$ denotes the Lebesgue measure.

Given a measurable payoff function $g$, the perpetual American option value $V$ in the jump diffusion model is defined by

$$
V=V(x, g)=\sup _{\tau} \mathrm{E}_{x}\left(\mathrm{e}^{-r \tau} g(X(\tau)) \mathbf{1}_{\{\tau<\infty\}}\right),
$$

where $\mathbf{1}_{\{\cdot\}}$ is the indicator function. Here the index indicates that $X(0)=x$, and the supremum is taken over all random times that are stopping times with respect to the filtration $\left(\mathcal{F}_{t}\right)_{0 \leq t<\infty}$.

Remark. When introducing jumps, completeness of the model is lost. Consequently there is a whole range of possible martingale measures that can be used for arbitrage-free pricing of options. We circumvent the issue of choosing a pricing measure by defining the option price as in (2.4), thus implicitly assuming that the dynamics of $X$ are specified directly under the measure used for pricing. 
We will only consider payoff functions $g$ in the class

$$
g:=\left\{g: \mathbb{R}^{+} \rightarrow[0, \infty) \text { with } g(x) \leq C(1+x) \text { for some constant } C>0\right\},
$$

i.e. payoff functions of at most linear growth. Since $\mathrm{e}^{-r t} X(t)$ is a nonnegative martingale, Problem I.3.16 and the Optional Sampling Theorem I.3.22 in Karatzas and Shreve (2000) imply that the American option price is finite for any $g \in g$.

Below we compare the option price $V$ in the jump diffusion model to the value $B S$ of a perpetual American option in the standard Black-Scholes model with a volatility $\gamma>0$, possibly different from $\sigma$. Recall that in the standard Black-Scholes model, the stock price $Y$ is modeled under the pricing measure by a geometric Brownian motion

$$
\mathrm{d} Y=r Y \mathrm{~d} t+\gamma Y \mathrm{~d} W .
$$

More explicitly,

$$
Y(t)=Y(0) \exp \left\{\left(r-\frac{\gamma^{2}}{2}\right) t+\gamma W(t)\right\} .
$$

If $g \in g$ is a payoff function, then the corresponding value $B S$ of a perpetual American option in the standard Black-Scholes model is

$$
B S=B S^{\gamma}(x, g)=\sup _{\tau} \mathrm{E}_{x}\left(\mathrm{e}^{-r \tau} g(Y(\tau)) \mathbf{1}_{\{\tau<\infty\}}\right) .
$$

Given a jump diffusion model, i.e. given $\sigma, \lambda$, and $\phi$, we are interested in determining a volatility $\gamma>0$ such that

$$
V(x, g) \leq B S^{\gamma}(x, g) \text { for all } x \text { and all payoff functions } g \in g .
$$

In Theorem 2.1, below, we prove the existence of a volatility $\gamma$ satisfying (2.5), and we also characterize the minimal such volatility as the smallest zero of the function $h:(0, \infty) \rightarrow$ $\mathbb{R} \cup\{\infty\}$ defined by

$$
h(\gamma):=\left(\frac{\sigma^{2}}{\gamma^{2}}-1\right) r\left(1+\frac{2 r}{\gamma^{2}}\right)+\lambda \int_{0}^{1}\left((1+\phi)^{-2 r / \gamma^{2}}-1+\frac{2 r}{\gamma^{2}} \phi\right) \mathrm{d} z .
$$

Lemma 2.1. The function $h:(0, \infty) \rightarrow \mathbb{R} \cup\{\infty\}$ is nonnegative on $(0, \sigma)$, and it satisfies $h(\gamma) \rightarrow-r$ as $\gamma \rightarrow \infty$. Moreover, $h$ is either

(i) finite for all $\gamma$,

(ii) equal to $\infty$ on $\left(0, \gamma^{\prime}\right)$ and finite on $\left[\gamma^{\prime}, \infty\right)$, for some $\gamma^{\prime} \in(0, \infty)$, or

(iii) equal to $\infty$ on $\left(0, \gamma^{\prime}\right]$ and finite on $\left(\gamma^{\prime}, \infty\right)$, for some $\gamma^{\prime} \in(0, \infty)$.

In case (iii) we have

$$
\lim _{\gamma \searrow \gamma^{\prime}} h(\gamma)=\infty
$$

Regardless of which class $h$ belongs to, it is continuous on the set where $h<\infty$.

Proof. It is clear that $h \geq 0$ on $(0, \sigma)$ since both terms are nonnegative (the integrand

$$
(1+\phi)^{-2 r / \gamma^{2}}-1+\frac{2 r}{\gamma^{2}} \phi
$$


is positive since $x \mapsto(1+x)^{-2 r / \gamma^{2}}$ is a convex function). Next, note that the integrand tends to 0 pointwise as $\gamma$ tends to $\infty$. By (2.3) and dominated convergence, the integral term tends to 0 and, consequently, $h(\gamma) \rightarrow-r$ as $\gamma \rightarrow \infty$. Moreover, from (2.2) it follows that the only way to have $h(\gamma)=\infty$ is if

$$
\int_{0}^{1}(1+\phi)^{-2 r / \gamma^{2}} \mathrm{~d} z=\infty .
$$

Thus, it follows that if $h(\gamma)=\infty$ then $h\left(\gamma^{\prime}\right)=\infty$ for all $\gamma^{\prime}<\gamma$. Consequently, $h$ has to belong to one of the classes (i)-(iii). Finally, $\lim _{\gamma \searrow \gamma^{\prime}} h(\gamma)=\infty$ in case (iii) follows from monotone convergence, and the continuity on the set where $h$ is finite follows from dominated convergence.

We now present our main result.

Theorem 2.1. Let a jump diffusion model be given as specified above. Then there exists a $\gamma>0$ such that (2.5) holds. If $\hat{\sigma}:=\inf \{\gamma>0:(2.5)$ holds $\}$ and $\hat{\gamma}:=\inf \{\gamma>0: h(\gamma) \leq 0\}$, then $\hat{\sigma}=\hat{\gamma}$ and

$$
V(x, g) \leq B S^{\hat{\gamma}}(x, g),
$$

for all $x$ and all payoff functions $g$. Moreover, $\hat{\gamma} \geq \sigma$.

Proof. Since $h(\infty):=\lim _{\gamma \rightarrow \infty} h(\gamma)=-r$ (cf. Lemma 2.1), there clearly exists a $\gamma>0$ with $h(\gamma) \leq 0$. Let such a $\gamma$ be given, and let $\varphi(x):=x^{-2 r / \gamma^{2}}$. Note that

$$
\mathcal{A} \varphi(x)=h(\gamma) \varphi(x)
$$

where $\mathcal{A}$ is the integro-differential operator

$$
\begin{aligned}
\mathcal{A} \varphi(x):= & \frac{\sigma^{2} x^{2}}{2} \varphi_{x x}(x)+r x \varphi_{x}(x)-r \varphi(x) \\
& +\lambda \int_{0}^{1}\left(\varphi(x+\phi x)-\varphi(x)-\phi x \varphi_{x}(x)\right) \mathrm{d} z
\end{aligned}
$$

associated with the jump diffusion $X$ (here the subindices denote differentiation with respect to $x)$. Consequently, if $h(\gamma) \leq 0$ then the process $\mathrm{e}^{-r t} \varphi(X(t))$ is a supermartingale, so optional sampling yields

$$
\mathrm{E}_{x}\left(\mathrm{e}^{-r \tau} \varphi(X(\tau)) \mathbf{1}_{\{\tau<\infty\}}\right) \leq \varphi(x),
$$

for any stopping time $\tau$. Now, define the strictly increasing function $F:(0, \infty) \rightarrow(0, \infty)$ by

$$
F(x):=\frac{x}{\varphi(x)}=x^{1+2 r / \gamma^{2}} .
$$

We now claim that, for any function $U:(0, \infty) \rightarrow[0, \infty)$, the implication

$$
\left\{U \geq g \text { and the composed function }(U / \varphi) \circ F^{-1} \text { is concave }\right\} \Longrightarrow U \geq V
$$

holds. To see this we argue as in Dayanik and Karatzas (2003). First, fix a function $U$ with $U \geq g$ and such that $(U / \varphi) \circ F^{-1}$ is concave, and fix an initial point $x \in(0, \infty)$. Because of concavity, there exists an affine transformation $L:=c_{1} F+c_{2}$ such that $L(\cdot) \geq U(\cdot) / \varphi(\cdot)$ 
and $L(x)=U(x) / \varphi(x)$. Note that the constants $c_{1}$ and $c_{2}$ are both nonnegative since $U / \varphi$ is nonnegative on $(0, \infty)$. Consequently, for any stopping time $\tau$ we have

$$
\begin{aligned}
\mathrm{E}_{x}\left(\mathrm{e}^{-r \tau} g(X(\tau)) \mathbf{1}_{\{\tau<\infty\}}\right) & \leq \mathrm{E}_{x}\left(\mathrm{e}^{-r \tau} U(X(\tau)) \mathbf{1}_{\{\tau<\infty\}}\right) \\
& \leq \mathrm{E}_{x}\left(\mathrm{e}^{-r \tau} \varphi(X(\tau)) L(X(\tau)) \mathbf{1}_{\{\tau<\infty\}}\right) \\
& =c_{1} \mathrm{E}_{x}\left(\mathrm{e}^{-r \tau} X(\tau) \mathbf{1}_{\{\tau<\infty\}}\right)+c_{2} \mathrm{E}_{x}\left(\mathrm{e}^{-r \tau} \varphi(X(\tau)) \mathbf{1}_{\{\tau<\infty\}}\right) \\
& \leq c_{1} x+c_{2} \varphi(x) \\
& =\varphi(x) L(x) \\
& =U(x),
\end{aligned}
$$

where we used (2.7) in the last inequality. Thus,

$$
V(x)=\sup _{\tau} \mathrm{E}_{x}\left(\mathrm{e}^{-r \tau} g(X(\tau)) \mathbf{1}_{\{\tau<\infty\}}\right) \leq U(x),
$$

so the claim (2.8) is proved.

Now note that $\varphi(x)=x^{-2 r / \gamma^{2}}$ and $\psi(x)=x$ are, respectively, the decreasing fundamental solution and the increasing fundamental solution of

$$
\frac{\gamma^{2} x^{2}}{2} u_{x x}+r x u_{x}-r u=0
$$

(see, for example, Chapter II of Borodin and Salminen (2002)). Recall from Dayanik and Karatzas (2003) that the smallest function $U:(0, \infty) \rightarrow[0, \infty)$ which satisfies $U \geq g$ and is such that $U / \varphi$ is $F$-concave is equal to the value $B S(\cdot, \gamma)$ of the American option in the Black-Scholes model with volatility $\gamma$. Thus, it follows from $(2.8)$ that $V(\cdot, g) \leq B S^{\gamma}(\cdot, g)$, which proves the existence of a $\gamma$ for which (2.5) holds.

By continuity of the function $h$ we clearly have $h(\hat{\gamma}) \leq 0$ (with equality in cases (i) and (iii) of Lemma 2.1). It follows from the above that $\hat{\sigma} \leq \hat{\gamma}$. To show that $\hat{\sigma} \geq \hat{\gamma}$, choose $\gamma \in(0, \hat{\gamma})$. Then $h(\gamma)>0$. For $C>0$, defining $g_{C} \in \mathcal{G}$ by

$$
g_{C}(x):=x^{-2 r / \gamma^{2}} \wedge C
$$

yields

$$
\left(\frac{\gamma^{2} x^{2}}{2} \partial_{x}^{2}+r x \partial_{x}-r\right) g_{C}(x) \leq 0
$$

(in the distributional sense). Consequently, $g_{C}$ is excessive for the geometric Brownian motion $Y$ with volatility $\gamma$, so $B S^{\gamma}\left(\cdot, g_{C}\right)=g_{C}(\cdot)$. On the other hand, for any fixed $x>0$ we have, for large $C$,

$$
\mathcal{A} g_{C}(x)=h(\gamma) x^{-2 r / \gamma^{2}}-\lambda \int_{0}^{1}\left((x+\phi x)^{-2 r / \gamma^{2}}-C\right) \mathbf{1}_{\left\{z:(x+\phi x)^{-2 r / \gamma^{2}} \geq C\right\}} \mathrm{d} z
$$

if $h(\gamma)<\infty$, which converges to $h(\gamma) x^{-2 r / \gamma^{2}}>0$ as $C$ tends to infinity. Consequently, $C$ can be chosen so that $\mathscr{A} g_{C}>0$ in some interval. (Similarly, it is easy to see that if $h(\gamma)=\infty$ then $C$ can be chosen large enough so that $\mathcal{A}_{C}>0$ in an interval.) This implies that $V>g_{C}$ in this interval, so there exist points $x$ with $V\left(x, g_{C}\right)>B S^{\gamma}\left(x, g_{C}\right)$. Since $\gamma \in(0, \hat{\gamma})$ is arbitrary, we find that $\hat{\sigma} \geq \hat{\gamma}$. This finishes the proof of $\hat{\sigma}=\hat{\gamma}$.

Finally, it is straightforward to check that $h(\gamma)>0$ for all $\gamma<\sigma$, which implies that $\hat{\gamma} \geq \sigma$. 
Remark. Note that the proof of Theorem 2.1 shows that $h(\hat{\gamma})=0$ if we are in case (i) or (iii) of Lemma 2.1, and $h(\hat{\gamma}) \leq 0$ if we are in case (ii). Moreover, since the Black-Scholes price is monotonically increasing in the volatility (see Alvarez (2003)), it follows from the proof of Theorem 2.1 that the function $h$ has at most one zero. More precisely, $h$ has precisely one zero (namely $\hat{\gamma}$ ) in cases (i) and (iii) of Lemma 2.1, and it has at most one zero in case (ii).

Theorem 2.1 provides an upper bound for American option prices. A lower bound can also be obtained for the class of convex contract functions.

Corollary 2.1. Let $g \in g$ be convex. Then

$$
B S^{\sigma}(x, g) \leq V(x, g) \leq B S^{\hat{\gamma}}(x, g),
$$

for all $x \in(0, \infty)$.

Proof. To prove the lower bound, note that the corresponding result for European options holds (see Theorem 4.1 of Bellamy and Jeanblanc (2000) or Theorem 5.1 of Ekström and Tysk (2005)). By approximating the American option with a sequence of Bermudan options, the bound carries over to our setting. The approximation details work as in Ekström (2004) or Ekström and Tysk (2005), and are therefore omitted.

Remark. We emphasize that the bounds provided in Corollary 2.1 hold for all (convex) payoff functions $g$. If studying a particular option, for example the put option with payoff function $g(x)=(K-x)^{+}$, then typically tighter bounds can be determined.

\section{Sensitivity of the minimal volatility with respect to the jump diffusion parameters}

It is natural to expect the minimal volatility $\hat{\gamma}$ to be large if the jump diffusion model in some sense is far away from a Black-Scholes model, i.e. if the jump intensity $\lambda$ and the possible relative jump sizes $\phi$ are large. Our next result shows that $\hat{\gamma}$ is increasing as a function of $\lambda, \phi$, and $\sigma$.

Theorem 3.1. Let two jump diffusion models be given with parameters $\left(\sigma_{1}, \lambda_{1}, \phi_{1}\right)$ and $\left(\sigma_{2}, \lambda_{2}, \phi_{2}\right)$. Assume that $\sigma_{1} \leq \sigma_{2}, \lambda_{1} \leq \lambda_{2}$, and

$$
\frac{\phi_{2}(z)}{\phi_{1}(z)} \geq 1
$$

for all $z$ with $\phi_{1}(z) \neq 0$. Then the corresponding minimal volatilities $\hat{\gamma}_{1}$ and $\hat{\gamma}_{2}$ satisfy $\hat{\gamma}_{1} \leq \hat{\gamma}_{2}$.

Proof. It is straightforward to check that the function $h(\gamma)=h\left(\gamma, \sigma^{2}, \lambda, \phi\right)$ defined in (2.6) is increasing in $\sigma^{2}$ and $\lambda$. Indeed,

$$
\frac{\partial}{\partial \sigma^{2}} h\left(\gamma, \sigma^{2}, \lambda, \phi\right)=\frac{\gamma^{2} r+2 r^{2}}{\gamma^{4}} \geq 0
$$

and

$$
\frac{\partial}{\partial \lambda} h\left(\gamma, \sigma^{2}, \lambda, \phi\right)=\int_{0}^{1}\left((1+\phi)^{-2 r / \gamma^{2}}-1+\frac{2 r}{\gamma^{2}} \phi\right) \mathrm{d} z \geq 0 .
$$

This implies that $\hat{\gamma}$, being the smallest solution $\gamma>0$ of $h(\gamma) \leq 0$, is increasing in $\lambda$ and $\sigma$. Moreover, the function

$$
g(a):=(1+a)^{-2 r / \gamma^{2}}-1+\frac{2 r}{\gamma^{2}} a
$$


satisfies

$$
\frac{\mathrm{d}}{\mathrm{d} a} g(a)=\frac{2 r}{\gamma^{2}}\left(1-(1+a)^{-1-2 r / \gamma^{2}}\right),
$$

which is positive if $a$ is positive and negative if $a$ is negative. Consequently, the function $h$ is increasing in $\phi$ if different $\phi$ are ordered as in the formulation of the theorem. It follows that $\hat{\gamma}$ is increasing also in $\phi$.

Similarly, if the model is close to the Black-Scholes model, i.e. if the intensity $\lambda$ of jumps is small, then we expect the minimal volatility $\hat{\gamma}$ to be close to $\sigma$.

Theorem 3.2. Assume that (2.3) holds with $\varepsilon=-2 r / \sigma^{2}$. Then, for small intensities $\lambda$, the quantity $\hat{\gamma}^{2}-\sigma^{2}$ is at most linear in $\lambda$. More explicitly, there exist constants $M>0$ and $\lambda_{0}>0$ such that $0 \leq \hat{\gamma}^{2}-\sigma^{2} \leq M \lambda$ for $\lambda \in\left[0, \lambda_{0}\right]$.

Proof. Let

$$
C:=\int_{0}^{1}\left((1+\phi)^{-2 r / \sigma^{2}}-1+\frac{2 r}{\sigma^{2}} \phi\right) \mathrm{d} z .
$$

It is straightforward to check that if $\lambda C \leq r$ and $\gamma^{2}=\sigma^{2}+\sigma^{2} \lambda C /(r-\lambda C)$, then

$$
\begin{aligned}
h(\gamma) & =\left(\frac{\sigma^{2}}{\gamma^{2}}-1\right) r\left(1+\frac{2 r}{\gamma^{2}}\right)+\lambda \int_{0}^{1}\left((1+\phi)^{-2 r / \gamma^{2}}-1+\frac{2 r}{\gamma^{2}} \phi\right) \mathrm{d} z \\
& \leq\left(\frac{\sigma^{2}}{\gamma^{2}}-1\right) r\left(1+\frac{2 r}{\gamma^{2}}\right)+\lambda C \\
& \leq 0
\end{aligned}
$$

Consequently, $\hat{\gamma}^{2} \leq \sigma^{2}+\sigma^{2} \lambda C /(r-\lambda C)$ for small intensities $\lambda$, which finishes the proof.

\section{References}

Alvarez, L. H. R. (2003). On the properties of $r$-excessive mappings for a class of diffusions. Ann. Appl. Prob. 13, 1517-1533.

Bellamy, N. And Jeanblanc, M. (2000). Incompleteness of markets driven by a mixed diffusion. Finance Stoch. 4, 209-222.

Borodin, A. N. And Salminen, P. (2002). Handbook of Brownian Motion. Facts and Formulae, 2nd edn. Birkhäuser, Basel.

Dayanik, S. And Karatzas, I. (2003). On the optimal stopping problem for one-dimensional diffusions. Stoch. Process. Appl. 107, 173-212.

Ekström, E. (2004). Properties of American option prices. Stoch. Process. Appl. 114, 265-278.

EKström, E. AND TysK, J. (2005). Properties of option prices in models with jumps. Submitted.

Karatzas, I. And Shreve, S. (2000). Brownian Motion and Stochastic Calculus, 2nd edn. Springer, Berlin.

McKean, H. P. (1965). A free-boundary problem for the heat equation arising from a problem in mathematical economics. Industr. Manag. Rev. 6, 32-39.

MoRdecKi, E. (2002). Optimal stopping and perpetual options for Lévy processes. Finance Stoch. 6, $473-493$. 